\title{
USO DA AURICULOTERAPIA NA ATENÇÃO PRIMÁRIA À SAÚDE: RELATO DE EXPERIÊNCIA
}

\section{USE OF AURICULOTHERAPY IN PRIMARY HEALTH CARE: EXPERIENCE REPORT}

\author{
Andreia da Silva Morais ${ }^{1}$ \\ Mariana Nunes Azevedo de Melo ${ }^{2}$ \\ Maria Júlia Galindo Soares ${ }^{3}$ \\ Paôlla Gabrielly Antas Lunguinho Dantas ${ }^{4}$ \\ Maria Adelaide Silva Paredes Moreira ${ }^{5}$ \\ Carolina Carvalho Nogueira ${ }^{6}$
}

RESUMO: Objetivo: Relatar a experiência do uso da auriculoterapia em um grupo de mulheres na atenção primária à saúde. Metodologia: É um estudo descritivo de natureza qualitativa na modalidade de relato de experiência, a partir de um Grupo de práticas integrativas e complementares na Unidade de Saúde da Família do Bessa João Pessoa, com cerca de 20 mulheres. O registro dos relatos das usuárias foi feito através de gravações que foram transcritas e analisadas. Resultados: A auriculoterapia foi utilizada no tratamento da ansiedade, estresse, dores, tabagismo, insônia, entre outros. Todas as mulheres relataram melhoras com a terapia, além de benefícios na qualidade de vida. Conclusão: $O$ uso da auriculoterapia na perspectiva das mulheres atendidas nesta Unidade de Saúde foi de grande importância e impacto, tendo em vista a sua resolubilidade e os resultados obtidos.

DESCRITORES: Auriculoterapia; atenção primária à saúde; estágio clínico; assistência Integral à saúde da mulher.

\footnotetext{
${ }^{1}$ Acadêmica do Curso de Fonoaudiologia da Universidade Federal da Paraíba, João Pessoa. E-mail: andreia.sm10@gmail.com.

${ }_{2}^{2}$ Acadêmica do Curso de Fonoaudiologia da Universidade Federal da Paraíba, João Pessoa. E-mail: mariananadm@gmail.com.

${ }^{3}$ Acadêmica do Curso de Fonoaudiologia da Universidade Federal da Paraíba, João Pessoa. E-mail: juliagalindo8@gmail.com.

${ }^{4}$ Acadêmica do Curso de Fonoaudiologia da Universidade Federal da Paraíba, João Pessoa. E-mail: paollagabrielly19@gmail.com.

${ }^{5}$ Professora Doutora do Departamento de Fonoaudiologia da Universidade Federal da Paraíba, João Pessoa. E-mail: jpadelaide@hotmail.com.

6onoaudióloga formada na UNIPÊ - Centro Universitário de João Pessoa. E-mail: fgacarolina@gmail.com.
} 
ABSTRACT: Objective: To register an experience of using auriculotherapy in a group of women with primary health care. Methodology: It is a descriptive study of qualitative nature in the modality of experience, from a Group of integrative and complementary practices in the Family Health Unit Bessa - João Pessoa, with about 20 women. The recording of user reports was made through recordings that were transcribed and analyzed. Results: Auriculotherapy was used to treat anxiety, stress, pain, smoking, insomnia, among others. All women relate better to therapy, in addition to benefits in quality of life. Conclusion: The use of auriculotherapy from the perspective of women treated at this Health Unit was of great importance and impact, in view of its resolution and the results obtained.

KEYWORDS: Auriculotherapy; primary health care, clinical clerkship, comprehensive health care for women. 


\section{INTRODUÇÃO}

A atenção primária à saúde (APS) é caracterizada como o primeiro nível do sistema de serviços de saúde, sendo a porta de entrada preferencial do usuário, solucionando os problemas de saúde com ações resolutivas, se articulando com os demais níveis de complexidade, e formando uma rede integrada de serviços (STARFIELD, 2002).

As práticas integrativas no Sistema Único de Saúde (SUS) tem origem em 1970, com a Primeira Conferência Internacional de Assistência Primária em SaúdeAlma Ata, difundindo-se por todo o mundo, e no Brasil o movimento ganhou força a partir da Oitava Conferência Nacional de Saúde, que ocorreu em 1986. Depois de Alma Ata a Organização Mundial de Saúde (OMS) criou o Programa de Medicina Tradicional, que tinha como objetivo formular políticas com intuito de defender os conhecimentos tradicionais em saúde e os das Medicinas Complementares e Alternativas (TELESI -JÚNIOR, 2016).

O campo das práticas integrativas, alternativas ou complementares em saúde está em crescente visibilidade no Brasil, tendo em vista que esses recursos são utilizados em clínicas particulares, comunidades tradicionais, igrejas, movimentos sociais e entidades não governamentais. Assim, muitos estudos começaram a investigar e validar essas práticas e métodos, o que possibilitou que o Ministério da Saúde implementar regulamentações que estimulam a difusão da Medicina Complementar (ANDRADE; COSTA, 2010).

Em 2006, foi estabelecido no SUS a Política Nacional de Práticas Integrativas e Complementares, que apresenta como uma das suas prioridades a inserção e o fortalecimento das práticas integrativas e complementares no nível primário de atenção à saúde, com a explicação dos instrumentos, técnicas e práticas terapêuticas a serem implementadas no SUS.

No entanto, essas práticas encontram diversas dificuldades para ser implementadas, dentre elas, a insuficiência de dados de produção científica, as 
limitações no controle das práticas e a falta de especialistas e, por conseguinte, a carência de informação (SOUSA et al., 2012).

As Terapias alternativas, também nomeadas como Complementares e/ou Integrativas, são denominadas pela Organização Mundial da Saúde - OMS como Medicina Tradicional e compreendem um grupo de práticas de atenção à saúde não alopáticas que englobam atividades como a acupuntura, naturopatia, fitoterapia, meditação, reiki, terapia floral, auriculoterapia, entre outras. Estas terapias procuram atender ao indivíduo de forma holística, considerando o indivíduo na sua dimensão global sem perder de vista a sua singularidade, baseado na confiança e no vínculo terapeuta e usuário (BRASIL, 2006).

É importante destacar que os recursos utilizados nas Medicinas Complementares e Alternativas têm como objetivo prevenir os agravos e a recuperação da saúde através de tecnologias eficazes e seguras, estimulando mecanismos naturais, como uma escuta acolhedora, desenvolvimento de vínculo terapêutico e a integração do ser humano com o meio ambiente e a sociedade (TELESI-JÚNIOR, 2016).

As crenças místicas e espirituais se confirmam quando há a observação física da melhora da doença com o tratamento utilizado. As diversas metáforas sobre opostos auxiliaram na compreensão da doença de acordo com a visão chinesa, em que o homem representa o microcosmo que está dentro do universo, que seria o macrocosmo. Quando um indivíduo relata sentir alguma dor, essa é associada ao desequilíbrio do Qi. O Qi é uma energia que flui através de canais ao longo do corpo, chamados de "meridianos" e se dizia que a circulação dessa energia invisível era facilitada através da inserção de agulhas nos acupontos (OLESON, 2005).

Foi visto que não podia identificar a orelha como um órgão isolado, e que na realidade ela fazia ligações com todos os órgãos do corpo humano. O exame que era realizado na orelha tinha o objetivo de visualizar possíveis aparições de doenças a fim de poder curá-las (OLESON, 2005). A auriculoterapia caracteriza-se como "terapia pela aurícula", que é uma técnica de diagnóstico e de tratamento onde o pavilhão auricular é estimulado, essa terapia tem como compreensão simbólica o fato de que a orelha simboliza um feto de cabeça para baixo, sendo então considerada uma zona reflexa. O seu uso é mais adequado quando o estímulo for 
de natureza não invasiva, com o uso de sementes de mostarda (Vaccaria), esféricas metálicas, esferas de cristal, apongs, pastilhas de óxido de silício, entre outros (SILVÉRIO-LOPES; SEROISKA, 2013).

Dentre algumas patologias que são tratadas com a auriculoterapia, destacase a cefaleia/enxaqueca, lesões por esforço repetitivo, lombociatalgia, artrite/artrose, algias em ombros, fibromialgia, dismenorreia e disfunção temporomandibular (DTM). No entanto ainda é observado que em algumas situações clínicas somente a auriculoterapia não é suficiente. Um dos motivos que influencia o tratamento com essa prática é a falta de uniformidade na abordagem do processo, como por exemplo, a localização e a composição dos pontos que são estimulados (SILVÉRIOLOPES; SEROISKA , 2013).

Partindo do pressuposto que é a partir do relato de usuários que utilizam auriculoterapia que é possível inferir se o uso desta terapia contribui para redução de sintomas e, por conseguinte, para uma melhor qualidade de vida, o objetivo do estudo é relatar a experiência do uso da auriculoterapia em um grupo de mulheres na atenção primária à saúde.

\section{METODOLOGIA}

Trata-se de um estudo descritivo de natureza qualitativa na modalidade de relato de experiência, a partir da vivência com um grupo de mulheres que recebem auriculoterapia na Unidade de Saúde do Bessa, em João Pessoa-PB. São descritos alguns sintomas e patologias que as mulheres apresentaram, além de relatos sobre como se sentiram pós-sessão da aurículoterapia, além de descrever como essas atividades são realizadas. Este estudo fundamenta-se na literatura acerca das práticas integrativas, principalmente, da auriculoterapia e, também, das atividades realizadas em grupo na atenção primária à saúde.

O projeto do Grupo de práticas integrativas e complementares é realizado na sala de reuniões, que se localiza dentro da USF do Bessa. Os encontros acontecem toda semana, na quarta-feira. O atendimento com o uso de práticas integrativas 
iniciou em maio de 2019, implantado pela fonoaudióloga e gerente da Unidade. Aproximadamente entre quinze a vinte mulheres fazem parte do grupo. É importante destacar que a cada sessão esse número pode mudar, tendo em vista que o critério de inclusão, os quais são: mulher; que demonstre interesse na terapia; e que participe semanalmente do grupo. Esse grupo recebe mulheres com vários diagnósticos e sintomas, dentre eles: transtorno da bipolaridade, câncer, dores de cabeça, dores no calcanhar, problemas emocionais e psicológicos, glaucoma, entre outros.

Para a sessão são utilizados os materiais básicos para a aplicação da auriculoterapia, sendo o álcool, para a limpeza do pavilhão auricular, semente de mostarda que será colocado nos pontos que vão ficar sendo estimulados no decorrer da semana e uma pinça que auxilia na aplicação da semente. A sala ainda conta com a imagem do pavilhão auricular e os respectivos pontos que podem ser estimulados de acordo com a queixa da paciente.

Cada sessão tem início com uma conversa em grupo, e logo depois cada mulher que integrante do grupo relata quais são os seus sintomas e respondem perguntas diretas feitas pela fonoaudióloga. Nessa conversa as mulheres também apontaram os pontos positivos e negativos da auriculoterapia, além de relatar se houve alguma melhora comparando com a semana anterior. Os relatos foram registrados na sessão do dia 12 de dezembro de 2019, por meio de uma gravação em áudio do celular ASUS Max shot. A cada usuária foi perguntado "Quais os pontos positivos e negativos após as sessões de auriculoterapia?" e a respostas delas foram gravadas.

Os encontros para a aplicação da auriculoterapia acontecem todas às quartas-feiras. Devido à dinâmica de fluxo continuo do grupo não é possível registrar o número preciso de sessões de cada mulher, mas a média é em torno de 28 sessões. 


\section{RESULTADOS}

O projeto do Grupo de Práticas Integrativas e Complementares foi idealizado pela fonoaudióloga e gerente da Unidade de Saúde do Bessa, que tem formação na técnica de auriculoterapia e que após observar uma demanda maior de mulheres que procuravam a unidade com diversas queixas de saúde, deu inicio ao grupo. Assim, em maio de 2019 iniciaram-se os atendimentos em grupo. O grupo é atualmente formado por 20 mulheres. A experiência é relatada a partir da importância e os resultados do uso de práticas integrativas na atenção primária à saúde.

$\mathrm{Na}$ atenção primária o trabalho de grupos é uma das possibilidades de práticas assistenciais. Nesses espaços pode ocorrer o aprimoramento dos indivíduos que estão envolvidos, através da valorização dos saberes e por meio da intervenção no processo de saúde-doença de cada usuário de forma criativa. Além disso, as atividades em grupos possibilita uma participação ativa do sujeito, atitudes, conhecimentos e habilidades para enfrentar os problemas de saúde (DIAS; SILVEIRA; WITT, 2009).

Um estudo realizado em Porto Alegre teve como objetivo explorar a organização das atividades de grupo nos seus locais de implantação. Participaram da pesquisa 124 unidades básicas de saúde e foi observado que em 96 unidades realizavam atividades de grupo, e que, em média, 4,03\% atividades em grupo com diferentes temáticas. Tendo em vista que são os níveis centrais da gestão que decidem estes grupos, de acordo com a situação de risco presente em determinadas frações populacionais (gestantes, crianças) ou, especificamente, a determinadas condições patológicas como, tuberculose, asma, hipertensão, diabetes (MAFFACCIOLLI; LOPES, 2011). No entanto, essas atividades preconizam a realização de diálogos, com enfoque na educação em saúde, não sendo relatado uso de práticas integrativas em nenhum grupo, o que difere da experiência relatada nesse estudo, onde além de abordar essas atividades citadas, também é realizado um atendimento com a auriculoterapia. 
As práticas integrativas e complementares são acima de tudo um novo modo de aprender e de praticar saúde. A crescente busca por essas práticas não se dá exclusivamente pelo fato de faltar opções médicas, mas sim pela busca de um atendimento mais humanizado e sensível para o paciente, sendo muito eficaz no que diz respeito à aprendizagem, a prática e ao cuidado da saúde tanto de si quanto dos demais envolvidos. Esse movimento traz novos olhares para a prática em saúde, gerando uma possível beleza em sua produção (TELESI- JUNIOR, 2016).

Estudos apontam que terapias em grupo servem para dar suporte aos indivíduos, e também como uma fonte de fortalecimento para os membros presentes. Quando o indivíduo tem a oportunidade de saber que outras pessoas passam por diversas questões, e algumas parecidas com a sua, há uma sensação de conforto e compreensão. É uma terapia diversificada, em que o indivíduo tem a oportunidade de conhecer diversas visões a respeito do seu problema e do problema do outro, havendo uma troca de experiência. (DUARTE; CARVALHO; BRENTANO, 2018), Esta afirmação está em consonância com o que foi observado no grupo de mulheres que recebem auriculoterapia, pois elas relatam que a troca de experiências e o momento de interação são de extrema importância para a vida e, por conseguinte, para a saúde.

A auriculoterapia é uma prática segura e de rápida aplicação, podendo ser realizada em diferentes condições locais e ambientais, em que o objetivo sempre é promover a qualidade de vida do sujeito (MOURA et al, 2014). Assim, a aplicação da auriculoterapia em 20 mulheres ocorreu nas quartas-feiras, em uma sala com cadeiras dispostas em circulo, com a presença da coordenadora, levando em media uma hora por semana. (Figura 1). 
Figura 1- Grupo de mulheres que recebem Auriculoterapia.

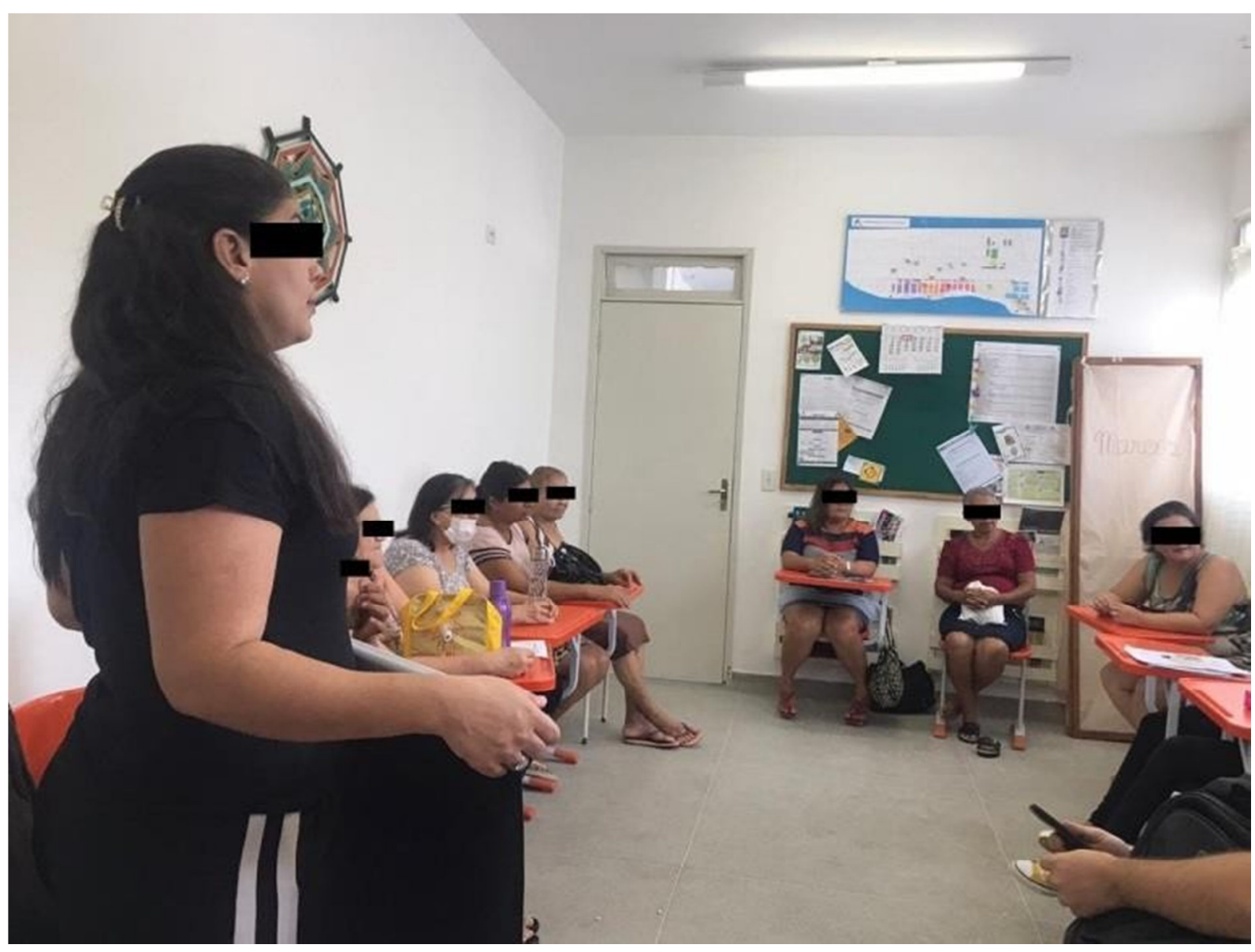

Fonte: Próprio autor.

A auriculoterapia também é utilizada para redução do grau de ansiedade em gestantes, sendo observado que a efetividade é dada devida o controle da dor, e consequentemente a redução da ansiedade. Essa redução da ansiedade também foi observada no momento do trabalho de parto das gestantes, onde normalmente é visto que esse nível aumenta (MEFETONI et al., 2018). A maioria das usuárias do grupo relatou que os estímulos da auriculoterapia reduzem as dores, como cefaleia, dores no calcanhar, do corpo, entre outras, e que isso ajuda a obter uma melhor qualidade de vida. Os relatos das usuárias apontam os benefícios advindos da aplicação da técnica, após o inicio das sessões. Relatos esses que se iniciaram a partir da primeira semana de uso da auriculoterapia, como podemos ver a seguir: 
[...] Bom é. minha ansiedade diminuiu bastante né? Quando você coloca no ponto do (incompreensível) onde você colocou, é. eu senti que diminuiu bastante, não cessou totalmente assim, a ponto de a parei [...] Ele diminui assim é, consideravelmente ele diminui, só que eu, ele não cessa, é no sentido de auxiliar mesmo, e isso pra mim foi muito bom porque me ajudou bastante, no sentido de combater a ansiedade, 0 hábito, a vontade de tá no hábito de fumar [...] Sim na questão das dores na coluna, é alivia bastante as dores na coluna, muito bom a sensação é de alívio imediato quando você coloca no ponto da coluna cervical. E esse aí não é auxiliar não ele está me curando mesmo porque eu não tomo nenhum remédio é muito raro. Usuária I - (Tabagista, ansiedade, dor cervical)

[...] Melhorou muito minha ansiedade e meu sono, melhora muito. Me sinto muito bem quando eu faço. A tendinite, melhorei muito da tendinite, nos dois ombros. Usuária II (ansiedade, insônia e tendinite)

Eu tenho problema né? No calcanhar, eu não identifiquei ainda, fiz exames, mas falta identificar ainda, também tenho problema de coluna muito sério, Devido às duas gestações que eu tive, e:: graça a Deus eu estou ótima sobre a coluna depois que eu usei durante esses dois meses né? Eu geralmente falo pra você o que ta me afetando e você tem colocado a auriculo e tem dado certo, e o resultado, a resposta é na mesma hora, imediato onde está doendo né? $\mathrm{Na}$ mesma hora para, é incrível, eu fico sem resposta para saber como que algo acontece tão rápido, sem precisar de medicações, isso é o mais importante. Usuária III - (dor, problema de coluna)

Em consonância com o relato da usuária I, que destacou a importância da auriculoterapia para o controle do tabagismo, uma pesquisa realizada com trabalhadores de uma universidade pública do sul de Minas Gerais, Brasil, observou que o tratamento com auriculoterapia contribuiu na redução do número de cigarros fumados para $61,9 \%$ dos participantes, além de destacar que o tabagismo não cessou completamente, podendo ser explicado devido parar de fumar ser um fenômeno complexo e multifatorial, que envolve aspectos biológicos, psicológicos e comportamentais (SILVA et al, 2014).

[...] Eu estou ainda, mas estava bem acima do peso e pedi para colocar em pontos que me ajudassem a controlar um pouco a 
ansiedade é, também com relação a alimentação porque eu comecei a fazer uma reeducação alimentar e a ansiedade atrapalha. O tempo que fiz me ajudou a controlar mais e me alimentar de forma mais adequada, em 7 meses eu já diminuí 9 $\mathrm{kg}$ e estou já perto de diminuir $10 \mathrm{~kg}$. $E$ também eu tenho muita dor no calcanhar porque eu tenho esporão, esporão de calcâneo, e em uma certa vez eu pedir para colocar nos pontos mas não disse para que era, e Carol foi aplicando, aplicando. $\mathrm{Na}$ outra semana eu voltei lá e perguntei um ponto específico que ela aplicou o que aquele ponto representava no corpo e ela falou que era o calcanhar e eu nossa que curioso porque eu tava sentindo muita dor no calcanhar e o ponto que você colocou quando eu apertava doía muito e eu achei assim, eu sei que os pontos quando dói é porque está ligado a uma parte do corpo que tá precisando de reequilibrar ou ta inflamado, alguma coisa assim e curiosamente você acertou no ponto do calcanhar e eu tô praticamente sem dores. E assim ela foi seguindo todas as semanas colocando para melhorar o calcanhar e hoje eu já não preciso mais, desinflamou completamente. Usuária IV - (sobrepeso; esporão de calcâneo)

[...] Uma melhora significativa que eu tive com auriculoterapia foi no quesito zumbido de ouvido, que eu sofro bastante principalmente quando eu tô estressada, e houve melhora significativa na minha vida, aliviou bastante o incômodo que causa o zumbido. Usuária V - (zumbido)

Estudos concluíram que a auriculoterapia é efetiva na redução do peso corporal (BONIZOL et al, 2016). A redução de peso com a ajuda da auriculoterapia também foi relatada por uma usuária do grupo (Usuária IV), sendo possível observar que a mesma relacionou a perda de peso com a redução da ansiedade.

Um dos sintomas que foram relatados pelas usuárias do grupo foi o zumbido, sintoma este que também foi observado em um estudo que verificou o efeito da acupuntura através de uma comparação pelo Tinnitus Handicap Inventory (THI) final e inicial, tendo como resultado a melhora dos aspectos relacionados ao zumbido. A acupuntura se diferencia da auriculoterapia pois há a introdução de agulhas nos pontos auriculares (VIANA et al, 2013). Esse resultado, assim como o relato da usuária $\mathrm{V}$, demonstra que as práticas integrativas e complementares, como a acupuntura e auriculoterapia, auxiliam na redução do zumbido.

Dentre as mulheres que recebem auriculoterapia, um delas realiza tratamento oncológico e destaca a importância da terapia para redução de dores e de aspectos 
psicológicos negativos, como ansiedade. Para o alívio das dores dos pacientes oncológicos, estudos relatam diversas intervenções eficientes, sendo destacadas algumas técnicas, como a aromaterapia e as técnicas de massagem, tendo em vista que após a utilização destas práticas os pacientes relataram uma melhora de forma instantânea. Podendo ser destacado, também, que os métodos não farmacológicos têm eficácia em diversas outras áreas, favorecendo tanto os pacientes como os cuidadores (ABREU et al., 2009).

É importante destacar, que além da efetividade do uso das práticas integrativas evidenciada pelos usuários, é de extrema necessidade que os profissionais da USF procurem formação nas Práticas Integrativas e Complementares (PIC). Tendo em vista que os profissionais que se mostram favoráveis as PIC no tratamento do indivíduo levam em consideração todas as suas dimensões. Por isso muitos profissionais relacionam essas práticas com a integralidade da atenção (ZOBOLI , 2014).

\section{CONCLUSÃO}

O objetivo desse estudo foi avaliar a efetividade do tratamento com auriculoterapia oferecido pela Unidade de Saúde, assim, foi visto o bom feedback das mulheres, em que todas relataram sentir melhoras das suas respectivas queixas. Desta forma, conclui-se que o uso da auriculoterapia na perspectiva das mulheres atendidas, foi de grande importância e impacto, tendo em vista a sua resolubilidade.

Os resultados deste estudo estão em consonância com a literatura, visto que é abordado que o uso das práticas integrativas e complementares são técnicas que procuram atender o indivíduo de forma holística e que propicia uma melhora na qualidade de vida. É de grande importância a continuidade de outras pesquisas para que cada vez se torne parte do conhecimento popular, quanto também para reafirmar a sua eficiência. 


\section{REFERÊNCIAS BIBLIOGRÁFICAS}

ABREU, M. et al. Manejo não farmacológico da dor em pacientes com câncer: revisão sistemática. Online Brazilian Journal of Nursing, v. 8, n. 1, 2009.

ANDRADE, J.; COSTA, L. Medicina complementar no SUS: práticas integrativas sob a luz da Antropologia médica. Saúde e Sociedade, v. 19, p. 497-508, 2010.

BOZINOL, W.; DE OLIVEIRA S.; VALIATTI, T.; DALCIN, M. Tratamento da obesidade com auriculoterapia: relato de casos. Amazônia: Science \& Health, v. 4, n.3, p. 19-24, 2016.

BRASIL. Ministério da Saúde. Secretaria de Atenção à Saúde. Departamento de Atenção Básica. Política Nacional de Práticas Integrativas e Complementares no SUS - PNPICSUS. Brasília: Ministério da Saúde, 2006.

MOURA, Caroline et al. Auriculoterapia efeito sobre a ansiedade. Rev Cubana Enfermer. Ciudad de la Habana, v. 30, n. 2, jun. 2014.

DIAS, V.; SILVEIRA, D.; WITT, R. Educação em saúde: protocolo para o trabalho de grupos em Atenção Primária à Saúde. Revista de APS, v. 12, n. 2, 2009.

DUARTE, M.; CARVALHO, J.; BRENTANO, V. Percepção dos familiares acerca do grupo de apoio realizado em uma unidade de internação psiquiátrica. Rev. Gaúcha Enferm., Porto Alegre, v. 39, p. 2017-0115, 2018.

MAFFACCIOLLI, R.; LOPES, M. Os grupos na atenção básica de saúde de Porto Alegre: usos e modos de intervenção terapêutica. Ciência \& Saúde Coletiva, v. 16, p. 973-982, 2011.

MAFETONI, Reginaldo et al. Effectiveness of auriculotherapy on anxiety during labor: a randomized clinical trial. Revista latino-americana de enfermagem, v. 26, 2018.

OLESON, Terry. Generalidades e historia de la auriculoterapia. In: Auriculoterapia: Sistemas chino y occidental de acupuntura auricular. 3 ed. Buenos Aires: Médica Panamericana, cap. 1 p. 1-16, 2008.

SILVÉRIO-LOPES, S.; SEROISKA, M. Auriculoterapia para analgesia. In: SILVÉRIOS - LOPES, Sandra. Analgesia por acupuntura. Curitiba: Omnipax, cap. 1 p. 1-22, 2013.

SILVA, R.; CHAVES, E.; PILLON,S.; SILVA, M.; MOREIRA, D.; IUNES, D. Contribuições da auriculoterapia na cessação do tabagismo: estudo piloto. Rev Esc Enferm USP. v.5, n.48, p. 883-90, 2014.

SOUSA,M. et al. Práticas integrativas e complementares: oferta e produção de atendimentos no SUS e em municípios selecionados. Cadernos de Saúde Pública, v. 28, p. 2143-2154, 2012.

STARFIELD, B. Atenção primária: equilíbrio entre necessidades de saúde, serviços e tecnologia. Brasília, DF: Unesco, 2002.

TELESI-JUNIOR, Emílio. Práticas integrativas e complementares em saúde, uma nova eficácia para o SUS. Estud. av., São Paulo, v. 30, n. 86, p. 99-112, Apr. 2016.

VIANA, Paula et al. Efeito da acupuntura na qualidade de vida de trabalhadores portadores de zumbido expostos a ruídos: um estudo piloto. Revista Brasileira de Medicina do Trabalho. V. 11, n. 2, p. 53-9, 2013. 
ZOBOLI, E. L. C. P. Papel das práticas complementares na compreensão dos profissionais da Atenção Básica: uma revisão sistemática. Revista da escola de enfermagem. São Paulo, p.189-196, 2014. 